\title{
Human immunodeficiency virus and cerebrovascular diseases - review
}

\author{
Seidu A. Richard ${ }^{1,2}$, John Tampouri², Marian Sackey ${ }^{3}$, Ahmed N. Zakariah ${ }^{4}$ \\ ${ }^{1}$ Department of Immunology, Jiangsu University, Zhenjiang, China \\ ${ }^{2}$ Department of Surgery, Volta Regional Hospital, Ho, Ghana \\ ${ }^{3}$ Department of Pharmacy, Volta Regional Hospital, Ho, Ghana \\ ${ }^{4}$ Ghana National Ambulance Service, Ministry of Health, Accra, Ghana
}

\begin{abstract}
Human immunodeficiency virus (HIV) is thought to increase the risk of cerebrovascular disease, although few data exist linking these two disease entities. The aetiology of vasculopathy in both adults and children with HIV remains unknown. However, it has been postulated that direct infection by HIV, immune complex deposition, and impaired regulation of the immune response are the likely causes. HIV-associated vasculopathy in the cerebral circulation encompasses several forms of arterial disease occurring in the absence of any cause other than HIV infection. It includes disease of extracranial large arteries, intracranial medium sized arteries with or without aneurysm formation and small vessel disease. The pathology appears to differ across vessel types, and the exact mechanism by which HIV induces vessel wall damage in each case remains uncertain. Furthermore, immunosuppression secondary to HIV can predispose to opportunistic infections of the central nervous system. These acquired immune deficiency syndrome (AIDS)-defining infections include toxoplasmosis, cryptococcal meningitis, tuberculosis, cytomegalovirus, and progressive multifocal leukoencephalopathy secondary to JC virus reactivation, which can also present as cerebrovascular-like manifestation in patients with HIV. Although several causative mechanisms have been proposed for the relationship between HIV infection and cerebrovascular diseases, including HIV-associated dyslipidaemia, endothelial dysfunction, inflammation, and hypercoagulability, the pathogenesis of HIV-vasculopathy is not completely understood. Beside the above pathological entities, HIV itself has been implicated in the pathogenesis of cerebrovascular diseases, either through direct invasion and vessel wall destruction or cytokine-mediated vascular remodelling.
\end{abstract}

HIV AIDS Rev 2017; 16, 4: 212-219

DOI: https://doi.org/10.5114/hivar.2017.71874

Key words: human immunodeficiency virus and stroke, ischaemic stroke, haemorrhagic stroke, stroke inflammation, neuroimaging, stroke in young.

\section{Introduction}

Recent studies report a higher risk of ischaemic strokes (IS) and intracranial haemorrhage (ICH) in the human immunodeficiency virus (HIV)-infected population, particularly among those who are severely immunocompromised [1-3]. Several possible mechanisms have been hypothesised to account for stroke in association with acquired immune deficiency syndrome (AIDS), including a prothrombotic state [4] or a covert HIV-induced vasculopathy $[5,6]$. The pathogenesis

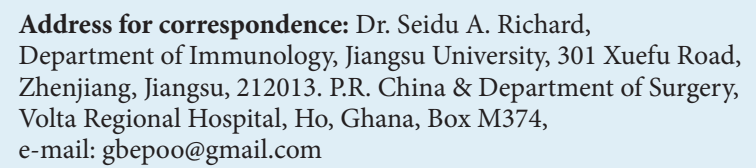

Address for correspondence: Dr. Seidu A. Richard, Department of Immunology, Jiangsu University, 301 Xuefu Road, Zhenjiang, Jiangsu, 212013. P.R. China \& Department of Surgery, Volta Regional Hospital, Ho, Ghana, Box M374, e-mail: gbepoo@gmail.com

Article history:

Received: 13.06.2017

Received in revised form: 29.07.2017

Accepted: 04.10.2017

Available online: 30.11 .2017
International Journal of HIV-Related Problems

HIV \& AIDS

R e vi e w 
of vasculopathy in both adults and children with HIV remains a matter of debate by scientists [7]. However, studies have mentioned the direct infection by HIV-1 virus, immune complex deposition, and impaired regulation of the immune response as likely causes [8]. While the strength of the associations and the pathogenic mechanisms remains a matter of debate, autopsy studies evaluating the cerebral vasculature in HIV-infected patients demonstrate a variety of pathologic changes. Small vessels appear to develop a vasculopathy, with perivascular space dilation, infarction, pigment deposition, vessel wall mineralisation, and perivascular cell infiltrates, all without evidence of a vasculitis [6], but recent studies have implicated inflammation as a course of vascular disease in the central nervous system (CNS) $[9,10]$. In larger cerebral vessels, progressive vessel dilation occurs in HIV infection, potentially mediated by a thinner media layer leading to aneurysm formation [11]. Additionally, there is evidence for increased arterial inflammation and carotid atherosclerosis in the setting of HIV infection $[9,10,12,13]$. These vascular changes could certainly disrupt cerebral autoregulation, diminish vessel wall tensile strength, and disrupt blood-brain barrier function, resulting in an increased risk of stroke. Although the biological mechanisms underlying the increased risk of ICH and IS in HIV-infected individuals likely overlap, there may be distinct mechanisms for each stroke subtype that are population-specific or manifest in the presence of other medical conditions and traditional stroke risk factors [14].

Furthermore, researchers have demonstrated that immunosuppression secondary to HIV can predispose to opportunistic infections in the CNS. These AIDS-defining infections include toxoplasmosis, cryptococcal meningitis, tuberculosis, cytomegalovirus, and progressive multifocal leukoencephalopathy (PML) secondary to JC virus reactivation, which can also present as cerebrovascular-like manifestation in HIV patients [15]. It is well known that HIV traverses the blood-brain barrier because the virus has been detected in the cerebrospinal fluid and brain parenchyma on brain biopsy [16], but the association between HIV and cerebrovascular diseases is still a matter of debate.

In view of several controversies associated with the pathogenesis of HIV and cerebrovascular diseases, we review published data on the association between HIV and CVDs. We looked at the association between varies types of stroke (ischaemic and haemorrhagic), vasculopathies, opportunistic infections leading to stroke in children, as well as radiological findings described in HIV and stroke patients. We used a universal search engine (Google Scholar) to search for scholarly publications on cerebrovascular diseases and HIV/ADIS.

\section{HIV opportunistic infections causing stroke}

Studies have indicated that vasculitic changes in intracerebral vessels associated with IS can be due to diverse opportunistic infections such as tuberculosis, cytomegalovirus infection, varicella-zoster infection, herpes simplex infection, syphilis, cryptococcosis, candidiasis, aspergillosis, mucormycosis, coccidioidomycosis, and trypanosomiasis [17]. Several cases of lymphomatoid granulomatosis and malignant lymphoma have also been associated with infarcts [17]. Stroke as a manifestation of HIV infection is poorly characterised $[18,19]$. This is primarily because most HIV patients presenting with a clinical picture suggestive of stroke are more likely to have an underlying focal or diffuse CNS infection or tumour responsible for their symptoms [19].

\section{HIV and CNS vasculopathy}

HIV-associated vasculopathy encompasses different clinical phenotypes, including large-vessel aneurysmatic disease and medium-vessel occlusive disease with or without fusiform dilation or stenosis (Fig. 1) [20]. HIV-associated cerebral vasculopathy was proposed as an important mechanism of stroke in the absence of a known cause, possibly related to deposition of a circulating immune complex or a direct toxic effect on the vascular endothelium [21]. The vascular histopathological changes in HIV-infected patients include medial fibrosis, loss of muscularis with destruction of the internal elastic lamina, and intimal hyperplasia [7]. Gutierrenz et al. in their study indicated that, patients with HIV infection have a thinner media to wall thickness ratio (MWR) than unaffected control subjects within the same age group; however, they excluded control subjects with different age groups [22]. Because none of the affected cases had a stroke, which suggests that the thinning of the media could be a preclinical stage in the occurrence of clinical CVDs in patients with HIV [22]. A thinner media has been reported in entities associated with arterial dilatation in patients with and without HIV [7, 20, 23, 24]. Some authors consider a more restrictively defined clinical syndrome, consisting of aneurysmal disease, termed aneurysmal vasculopathy [25].

Disruption of the internal elastic lamina (IEL) has been reported in HIV vasculopathy [23, 26], but this finding was not further investigated. Therefore, we propose further studies in this direction. Some authors suggest that IEL disruption is usually encountered in flow-mediated dilation [27]. The smooth muscle cells that form the media layer can be directly infected with HIV with progressive damage of the media with the aid of classical atherosclerosis and inflammation (Fig. 1) [28], although some endothelial role in the expression of metalloproteinases (MMPs) has also been suggested [29]. These findings suggest that in patients with HIV without clinical stroke, there is thinning of the media arterial layer, and thinning of the media could be a preclinical stage in the development of HIV vasculopathy. Guedes et al. describe an HIV+ patient with HIV-associated vasculopathy, who developed both IS and subarachnoid haemorrhage (SAH). Vasculopathy was imputed to CNS HIV compartmentalisation when treatment consisted of high-dose steroids followed by antiretroviral therapy (ART) adjustment to improve clinical penetration effectiveness (CPE) and 


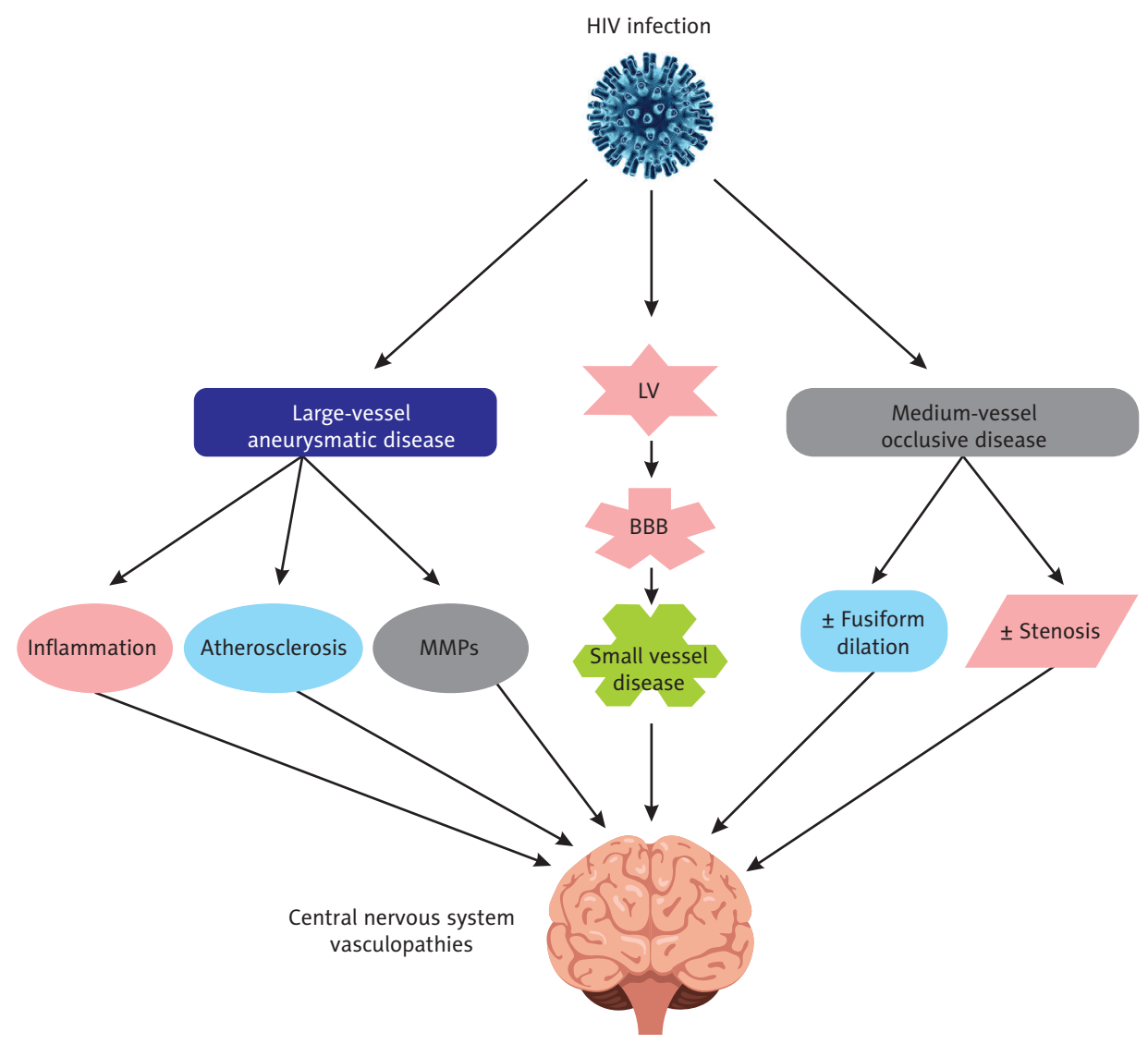

Fig. 1. HIV-associated vasculopathy encompasses large-vessel aneurysmatic disease and medium-vessel occlusive disease with or without fusiform dilation or stenosis. The large-vessel aneurysmatic disease occurs a result progressive HIV causing damage to the media accelerated by classical atherosclerosis, inflammation and metalloproteinases (MMPs) leading to vasculopathy. Medium-vessel occlusive disease also facilities by HIV with or without fusiform dilation or stenosis also leads to vasculopathy. Leukocytoclastic vasculitis (LV) of the vasa vasorum in extracranial carotid arteries and intimal abnormalities in intracranial medium sized vessels also leads to breakdown of the blood brain barrier (BBB) as a cause for small vessel disease leading to vasculopathy

vascular abnormalities improved alongside near-normalisation of CSF viral load on follow-up evaluation [30].

Studies have demonstrated leukocytoclastic vasculitis (LV) of the vasa vasorum in extracranial carotid arteries and intimal abnormalities in intracranial medium-sized vessels, and have hypothesised a breakdown of the blood brain barrier (BBB) as a cause for small vessel disease (Fig. 1) $[6,20,31]$. The vasculopathy may be asymptomatic and result in stroke, encephalopathy, cognitive impairment, or nonspecific neurological symptoms. Cutfield et al. advise a sensible approach to the management of HIV-associated vasculopathy with thorough investigation to exclude other infectious causes and autoimmune vasculitis [32]. Autopsy studies conducted by Connor et al. indicated that none of their cases showed evidence of vasculitis. Small-vessel occlusion was not observed, with the exception of one possible intravascular thrombus in a small penetrating artery in the basal ganglia of two cases and in a single neocortical penetrating artery in five cases. Fibrinoid necrosis of occasional neocor- tical and basal ganglia microvessels was a feature only in six cases; one brain was unusual in that it showed severe agonal global hypoxic-ischaemic damage [6].

\section{Inflammation as pathogenesis of stroke in HIV}

Although the sources of inflammatory cells in the adventitia of those with HIV is not clear, it is well known that the brain arteries are wrapped by the leptomeninges as they enter the skull, hence association with aseptic meningitis and adventitial inflammation seems plausible. It is now clear that inflammatory cells (macrophages and/or lymphocytes) are frequently reported in the arterial wall of in vitro and in vivo specimens with HIV with atherosclerosis [28, 33]. Researchers have also demonstrated concrete evidence of inflammation in HIV models of atherosclerosis (animal and human) and indicated that inflammation plays an important role in the pathophysiology of vascular disease. Further studies 


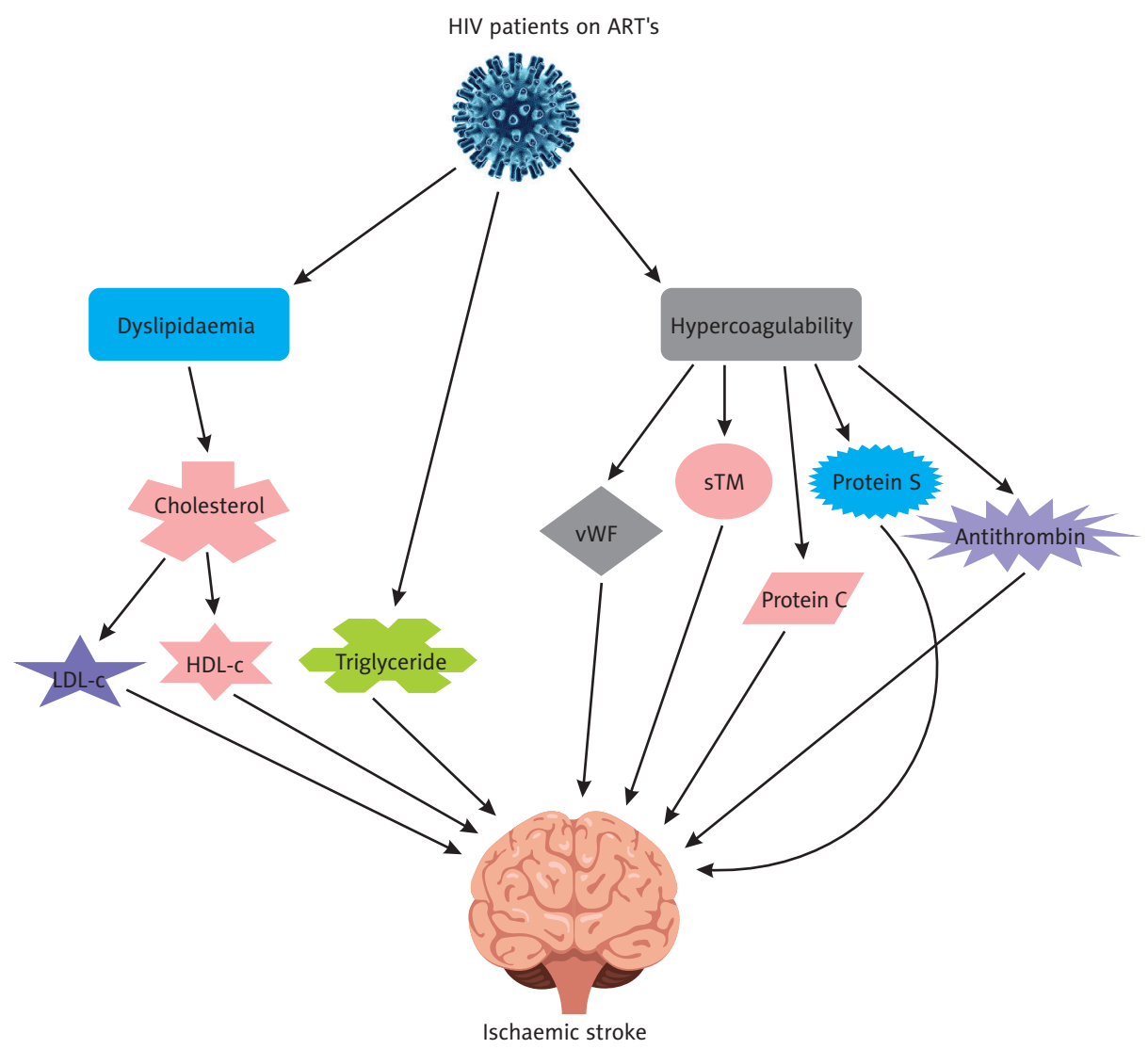

Fig. 2. HIV infection and cardiovascular risk including HIV-associated dyslipidaemia and hypercoagulability. HIV patients on antiretroviral therapy (ART) are associated with increases in total cholesterol (TC) and low-density lipoprotein cholesterol (LDL-c) but little change in high-density lipoprotein cholesterol (HDL-c), which are risk factors for ischaemic stroke. Plasma triglyceride levels are also high in HIV patients on or not on ARTs. They are elevated of plasma levels of endothelial cell products, including von Willebrand factor (VWF) and soluble thrombomodulin (STM) in those and deficiency of protein C and S as well as antithrombin in HIV patients. These factors all contribute to the development of ischaemic stroke

have indicated an increased expression of chemoattractant proteins in endothelial and other vascular cells (e.g. smooth muscle cells) upon exposure to HIV viral proteins like tat or p24 [28, 33, 34]. Studies have also indicated that HIV infection per se may contribute to cardiovascular disease (CVD) risk via nonspecific inflammation. Uncontrolled HIV infection is associated with elevated markers of inflammation, including C-reactive protein (CRP); levels of these markers decline with treatment but may or may not return to normal levels [35], and analysis from the FRAM study cohort has shown that fibrinogen and CRP are strong and independent predictors of mortality in HIV-infected adults even in those with relatively preserved CD4 counts $>500$ cells per microliter [36].

Gutierrez et al. in their study confirmed that HIV+ cases had a higher inflammation score than HIV- cases, but this difference was mostly driven by a significant difference in adventitial inflammation in HIV+ cases while intima inflammation was progressively more intense and widespread as the atherosclerotic phenotype evolved, but this effect was independent of HIV status. They indicated that inflammation in the media was typically observed in the context of intima inflammation [9]. Their study further indicated that in HIVcases as compared to HIV+ cases, intima inflammation was also the most important predictor of adventitial inflammation. They further argue that brain arteries in HIV+ cases appeared to have enhanced susceptibility to the development of adventitial inflammation compared with HIV- cases who have independent atherosclerosis or intimal inflammation. They therefore concluded that there is a strong association between HIV- and adventitial inflammation [9]. They also observed that contrary to what was seen in HIV- cases, adventitial inflammation in HIV+ cases were not entirely explained by associations with intimal inflammation because of atherosclerosis. It is also important to note that adventitial inflammation could be a predictor of dolichoectasia in arteries with a thin media, which supports the notion that HIV vasculopathy is a form of pathological brain arterial remodelling observed predominantly in HIV cases with persistent immunosuppression, in which adventitial inflammation is a surrogate of immunosuppression or a marker of the underlying pathophysiology leading to thinning of the media and subsequent arterial dilatation $[9,10]$. 


\section{HIV and ischaemic stroke}

Qureshi et al. in a retrospective case-control study indicated that HIV-1 infection was particularly associated with the occurrence of ischaemic stroke after adjustment of several cerebrovascular risk factors [37]. Studies have also indicated that the total prevalence rate of strokes in HIV patients is $1.9 \%$ with ischaemic stroke accounting for $1.2 \%$ whatever the age and the prevalence for juvenile ischaemic strokes occurring under the age of 46 years was 1.6\%, higher than in the HIV-negative population [38]. It is now clear that stroke patients are older, with lower CD4 cell count, and are in more advanced stages of the disease than infected patients without stroke [38]. Further studies have indicated that the majority of African American patients suffer ischaemic strokes, with small vessel occlusion being the most common stroke subtype followed by large vessel occlusion, cardio embolic, watershed, and cryptogenic [39]. Studies has further emphasized that HIV caused vasculitis of the carotid arteries in patients and this eventually led to the formation of hypo-echogenic plaques and consecutive stenosis $[40,41]$. Several causative mechanisms have been proposed for the relationship between HIV infection and cardiovascular risk including HIV-associated dyslipidaemia (Fig. 2), endothelial dysfunction (Fig. 1), inflammation (Fig. 1), and hypercoagulability (Fig. 2) [42].

HIV-infected patients tend to have decreases high-density lipoprotein cholesterol (HDL-c) and low-density lipoprotein cholesterol (LDL-c) levels, followed by an increase in plasma triglyceride levels prior to developing AIDS, and the degree of viremia may correlate with the amount of triglyceridaemia [42]. A prospective study of 50 men from the Multicentre AIDS Cohort Study has shown significant declines in mean serum total cholesterol (TC), HDL-c, and LDL-c [43], and subsequent initiation of ARTs was associated with increases in TC and LDL-c but little change in HDL-c (Fig. 2) (it is noteworthy to mention that TC and LDL-C were observed after about three years of ART, which possibly represents a return to pre-infection serum lipid levels after accounting for expected age-related changes) [42]. An increased pulse wave velocity (which may be an early marker for atherosclerosis) has been noted in two small studies of HIV-infected patients taking ARTs compared to HIVseronegative controls [44]. Endothelial function was assessed by brachial artery flow mediated dilation (FMD) in treatment-naive patients initiating ARTs with either a protease inhibitor (PI)-based, non-nucleoside reverse transcriptase inhibitor (NNRTI)-based, or nucleoside analogue-sparing regimen [45]. Rapid and significant improvement in FMD was seen in all treatment arms compared to baseline, suggesting that suppression of HIV and not the specific drugs themselves led to improved endothelial function $[45,46]$. However, antiretroviral medications may independently contribute to endothelial damage [42].

Otedo et al. presented eight cases of central nervous system and peripheral arteritis in HIV patients with low cluster of differentiation 4 (CD4) counts (2-200 per dl) [47]. Next to the HIV infection itself, treatment with highly active antiretroviral therapy, especially with protease inhibitors, may increase serum cholesterol and thus promote cerebrovascular disease in HIV-infected patients [48]. Several studies have also described elevated plasma levels of endothelial cell products, including von Willebrand factor (vWF) and soluble thrombomodulin (sTM) in those living with HIV (Fig. 2) [49]. sTM was strongly raised in those patients with the lowest CD4+ cell count, but levels of vWf increased at each incremental fall in CD4 cell count. A positive correlation has also been noted between HIV viral load and prothrombin fragment in $90 \mathrm{HIV}$-infected young individuals with CD4 cell count $216 \pm 198$ not receiving ARTs, suggesting that increasing levels of viraemia may increase the risk of thrombosis [50]. Furthermore research has shown the deficiency of protein $\mathrm{C}$ and S in HIV patients (Fig. 2) [51]. Although acute thrombosis lowers the levels of protein $\mathrm{C}$, protein $\mathrm{S}$, and antithrombin (Fig. 2) and the initiation of Warfarin lowers the levels of protein $\mathrm{C}$ and protein $\mathrm{S}$, normal values rule out those factors as risk factors for hypercoagulability in these patients [42].

\section{HIV and haemorrhagic stroke}

Research has indicated that ICHs are mainly localised in the subtentorial regions although their localisations can vary and are sometimes multiple. While small sized ICH could be asymptomatic, their mortality rate tends to be high [52]. A subdural haematoma was reported in an HIV-1-infected patient with an HIV-associated encephalopathy and cerebral atrophy [53]. However, intracerebral haemorrhage is a rare, late, and serious complication of AIDS with a mortality rate of $62.5 \%$ [54]. Some studies have indicated that haemorrhagic strokes accounted for $25 \%$ of all strokes and that comprise of intraparenchymal and subarachnoid [55]. While it is clear that vasculitis is usually found in small veins, cerebral venous thrombosis is very rare in HIV-1 infection [56]. It was noted that extensive intracranial sinus thrombosis was also the consequence of a hypercoagulable state complicating AIDSassociated nephrotic syndrome [57].

Studies have also indicated that cerebral arteriovenous malformation (AVM), although rare, might be considered in the differential diagnosis of acute cerebral haemorrhage in patients with HIV within the proper context, and prompt a proper diagnostic and therapeutic approach [58]. This was proposed by Alomari et al., who noted an unusual presentation of AVM in one case during their study. In attempts to explain the unusual presentation, they indicated that: 1) the presence of multiple cerebral AVMs is rare, 2) the occurrence of multiple episodes of cerebral haemorrhage secondary to multiple micro-AVMs has never been reported before in HIV, and 3) most of the cerebrovascular complications in the literature were reported in patients with advanced immunosuppression [58]. Their patient maintained a moderate immunosuppression status while receiving triple ART without signs of AIDS. They argue 
that in contrast to the multiple cerebral AVMs seen with hereditary haemorrhagic telangiectasia, which are superficially located within the cerebral cortex and are not associated with haemorrhage or venous pouches, the lesions encountered in their patient were deeply situated lesions within the cerebral parenchyma and developed multiple haemorrhages [58].

\section{HIV and stroke in children}

Park et al. described seven HIV+ children with clinical and/or neuroradiological evidence of stroke and estimated the clinical incidence of cerebrovascular events to be $1.3 \%$ per year [59]. Another study of $380 \mathrm{HIV}+$ children found that $1 \%$ had radiological evidence of a stroke [60]. In children, the risk is primarily related to coagulopathy, cardio embolism, vascular ectasia or aneurysms, subacute necrotising encephalopathy, and arteriopathy [61, 62]. Kure et al. demonstrated the presence of HIV within the arterial wall of the circle of Willis and in the cerebral parenchyma in a child who died of HIV-related cerebral vascular ectasia and multiple infarcts [63]. Schieffelin et al., with 51 subjects with diagnoses suggestive of CNS vasculopathy among the $3338 \mathrm{HIV}+$ subjects and after review, excluded 27 as not being consistent with CNS vasculopathy [64]. They indicated that despite being a rare event, the incidence rate of stroke is higher than the reported incidence rate of stroke in the general paediatric population, so they concluded that there is increased incidence of cerebrovascular events in HIV+ children, with the incidence among the HIV+ subjects being 2.4 times that of HEU subjects [64].

Whereas aneurysms of the great vessels can occur in the adult HIV population, CNS vasculopathy is more common in the paediatric population $[65,66]$. In paediatric HIV patients, aneurysms are characteristically diffuse, fusiform dilatations of the larger arteries around the Circle of Willis $[7,61]$. Some authors have suggested that the inflammation related to CNS vasculopathy, and aneurysm formation in particular, may result from coinfections in immunocompromised individuals $[67,68]$. The possibility that CNS vasculopathy results from an inflammatory response to HIV in the CNS has also been suggested $[63,69]$. There is evidence that HIV invades the CNS early in perinatal infection $[6,70]$. HIV DNA has been identified in the cerebral white matter and basal ganglia as well as in blood vessel walls $[60,71]$. A transient cerebral arteriopathy caused by a variety of infectious agents, including HIV, is found in children [72]. This typically presents with infarction in the territory of the lenticulostriate vessels associated with arteriopathy of the distal internal carotid artery, proximal middle cerebral artery, or anterior cerebral artery. The diagnosis requires follow-up imaging showing improvement in the arterial stenosis. However, transient cerebral arteriopathy has not been described in adults and is unlikely in these patients given the distribution of their brain lesions and protracted clinical course [73].

\section{Radiological findings of HIV patients with stroke}

Cole et al. with radiological studies demonstrated that AIDS is strongly associated with both IS and ICH although available angiographic investigations showed no evidence of vasculitis [14]. A study conducted with transcranial Doppler comparing HIV-infected patients and healthy control subjects excluding patients with pre-existing stenotic or occlusive lesions indicated that HIV-infected patients had reduced baseline cerebral blood flow and decreased cerebrovascular reserve capacity in response to acetazolamide challenge, suggesting altered vasoreactive responses in HIV infection [5]. Other studies are of the view that except for studies based on both clinical and pathological evidence, it is quite difficult to isolate strokes caused by intrinsic AIDS-associated pathophysiology in the absence of AIDS-related medical complications or other concomitant aetiologies [74]. This was reported in a population-based study to directly quantify the AIDSassociated risk of stroke, which attempted to address the possibility that pathophysiological mechanisms intrinsic to AIDS may predispose to stroke [74].

Further studies have indicated that in HIV patients presenting with acute stroke with corresponding neuroradiology findings, the most common lesions were segmental narrowing and dilatation of major cerebral arteries, basal ganglia infarction, and cortical atrophy [75]. Brain imaging studies have also shown right anterior corona radiata and basal ganglia acute infarction, more clearly shown on brain MRI with contrast [42]. Maggi et al. examined the ultrasonic structure of plaques in HIV-positive patients compared to those of atherosclerotic and inflammatory origins [76]. They found that carotid plaques of HIV-infected patients share several Duplex sonographic characteristics, e.g. the iso- or hypo-echogenic plaque morphology, with those found in carotid arteritis, and hence concluded that arteritis, but not arteriosclerosis, causes carotid artery disease and stroke in HIV infected patients [76].

\section{Conclusions}

Although several causative mechanisms have been proposed for the relationship between HIV infection and cerebrovascular diseases, including HIV-associated dyslipidaemia, endothelial dysfunction, inflammation, and hypercoagulability, the pathogenesis of HIV-vasculopathy is not completely understood. Besides all the pathological entities discussed above, HIV itself has been implicated in the pathogenesis of cerebrovascular diseases, either through direct invasion and vessel wall destruction or cytokine-mediated vascular remodelling. Despite sparse evidence on the role of vasculitis in the pathogenesis of HIV-associated vasculopathy, with few autopsy studies, HIV-particles or proteins have been demonstrated in vessel walls of a single paediatric patient, but some researchers still do not support this fact. In view of controversies in the association between the pathological association of HIV/ADIS and cerebrovas- 
cular disease, we noted a strong link between the two pathogenic processes. We propose further studies be conducted on some of the anti-retroviral medications because some them lead to vascular abnormalities.

\section{Conflict of interest}

The authors declare no potential conflicts of interest with respect to the research, authorship, and/or publication of this article.

\section{References}

1. Chow FC, Regan S, Feske S, et al. Comparison of ischemic stroke incidence in HIV-infected and non-HIV-infected patients in a US health care system. J Acquir Immune Defic Syndr 2012; 60: 351-358.

2. Ovbiagele B, Nath A. Increasing incidence of ischemic stroke in patients with HIV infection. Neurology 2011; 76: 444-450.

3. Rasmussen LD, Engsig FN, Christensen H, et al. Risk of cerebrovascular events in persons with and without HIV: a Danish nationwide population-based cohort study. AIDS 2011; 25: 1637-1646.

4. Hoffmann M, Berger JR, Nath A, et al. Cerebrovascular disease in young, HIV-infected, black Africans in the KwaZulu Natal province of South Africa. J Neurovirol 2000; 6: 229-236.

5. Brilla R, Nabavi DG, Schulte-Altedorneburg G, et al. Cerebral vasculopathy in HIV infection revealed by transcranial Doppler: a pilot study. Stroke 1999; 30: 811-813.

6. Connor M, Lammie G, Bell J, et al. Cerebral infarction in adult AIDS patients observations from the Edinburgh HIV Autopsy Cohort. Stroke 2000; 31: 2117-2126.

7. Dubrovsky T, Curless R, Scott G, et al. Cerebral aneurysmal arteriopathy in childhood AIDS. Neurology 1998; 51: 560-565.

8. Brannagan TH. Retroviral-associated vasculitis of the nervous system. Neurol Clin 1997; 15: 927-944.

9. Gutierrez J, Menshawy K, Gonzalez M, et al. Brain large artery inflammation associated with HIV and large artery remodeling. AIDS 2016; 30: 415-423.

10. Gutierrez J, Goldman J, Dwork AJ, et al. Brain arterial remodeling contribution to nonembolic brain infarcts in patients with HIV. Neurology 2015; 85: 1139-1145.

11. Gutierrez J, Elkind MS, Petito C, et al. The contribution of HIV infection to intracranial arterial remodeling: a pilot study. Neuropatho$\operatorname{logy} 2013 ; 33: 256-263$.

12. Kaplan RC, Kingsley LA, Gange SJ, et al. Low CD4+ T cell count as a major atherosclerosis risk factor in HIV-infected women and men. AIDS 2008; 22: 1615-1624.

13. Subramanian S, Tawakol A, Burdo TH, et al. Arterial inflammation in patients with HIV. JAMA 2012; 308: 379-386.

14. Cole JW, Chin JH. HIV infection: A new risk factor for intracerebral hemorrhage? Neurology 2014; 83: 1690-1691.

15. Tan IL, Smith BR, von Geldern G, et al. HIV-associated opportunistic infections of the CNS. Lancet Neurol 2012; 11: 605-617.

16. McRae M. HIV and viral protein effects on the blood brain barrier. Tissue Barriers 2016; 4: e1143543.

17. Rabinstein AA. Stroke in HIV-infected patients: a clinical perspective. Cerebrovasc Dis 2003; 15: 37-44.

18. Gray F, Gherardi R, Scaravilli F. The neuropathology of the acquired immune deficiency syndrome (AIDS). Brain 1988; 111: 245-266.

19. Pinto AN. AIDS and cerebrovascular disease. Stroke 1996; 27 : 538-543.

20. Tipping B, de Villiers L, Wainwright $\mathrm{H}$, et al. Stroke in patients with human immunodeficiency virus infection. J Neurol Neurosurg Psychiatry 2007; 78: 1320-1324.
21. Stapf C, Mohr JP, Pile-Spellman J, et al. Epidemiology and natural history of arteriovenous malformations. Neurosurgical Focus 2001; 11: 1-5.

22. Gutierrez J, Glenn M, Isaacson RS, et al. Thinning of the arterial media layer as a possible preclinical stage in HIV vasculopathy: a pilot study. Stroke 2012; 43: 1156-1158.

23. Chetty R, Batitang S, Nair R. Large artery vasculopathy in HIVpositive patients: another vasculitic enigma. Hum Pathol 2000; 31: 374-379.

24. Gutierrez J, Sacco RL, Wright CB. Dolichoectasia - an evolving arterial disease. Nat Rev Neurol 2011; 7: 41-50.

25. Goldstein DA, Timpone J, Cupps TR. HIV-associated intracranial aneurysmal vasculopathy in adults. J Rheumatol 2010; 37: 226-233.

26. Tipping B, de Villiers L, Candy S, et al. Stroke caused by human immunodeficiency virus-associated intracranial large-vessel aneurysmal vasculopathy. Arch Neurol 2006; 63: 1640-1642.

27. Masuda H, Zhuang Y-J, Singh TM, et al. Adaptive remodeling of internal elastic lamina and endothelial lining during flow-induced arterial enlargement. Arterioscler Thromb Vasc Biol 1999; 19: 22982307.

28. Eugenin EA, Morgello S, Klotman ME, et al. Human immunodeficiency virus (HIV) infects human arterial smooth muscle cells in vivo and in vitro: implications for the pathogenesis of HIV-mediated vascular disease. Am J Pathol 2008; 172: 1100-1111.

29. Toschi E, Barillari G, Sgadari C, et al. Activation of matrix-metalloproteinase-2 and membrane-type-1-matrix-metalloproteinase in endothelial cells and induction of vascular permeability in vivo by human immunodeficiency virus-1 Tat protein and basic fibroblast growth factor. Mol Biol Cell 2001; 12: 2934-2946.

30. Guedes BF, Gomes HR, Lucato LT, et al. Human immunodeficiency virus-associated vasculopathy with CNS compartmentalization of HIV-1. J Neurovirol 2015; 21: 101-104.

31. Modi G, Ranchod K, Modi M, et al. Human immunodeficiency virus associated intracranial aneurysms: report of three adult patients with an overview of the literature. J Neurol Neurosurg Psychiatry 2008; 79: $44-46$.

32. Cutfield NJ, Steele H, Wilhelm T, et al. Successful treatment of HIV associated cerebral vasculopathy with HAART. J Neurol Neurosurg Psychiatry 2009; 80: 936-937.

33. Tinkle BT, Ngo L, Luciw PA, et al. Human immunodeficiency virusassociated vasculopathy in transgenic mice. J Virol 1997; 71: 48094814.

34. Toborek M, Lee YW, Pu H, et al. HIV-Tat protein induces oxidative and inflammatory pathways in brain endothelium. J Neurochem 2003; 84: 169-179.

35. Triant VA, Meigs JB, Grinspoon SK. Association of C-reactive protein and HIV infection with acute myocardial infarction. J Acquir Immune Defic Syndr 2009; 51: 268-273.

36. Tien PC, Choi AI, Zolopa AR, et al. Inflammation and mortality in HIV-infected adults: Analysis of the FRAM study cohort. J Acquir Immune Defic Syndr 2010; 55: 316-322.

37. Qureshi AI, Janssen RS, Karon JM, et al. Human immunodeficiency virus infection and stroke in young patients. Arch Neurol 1997; 54: 1150-1153.

38. Evers S, Nabavi D, Rahmann A, et al. Ischaemic cerebrovascular events in HIV infection. Cerebrovasc Dis 2003; 15: 199-205.

39. Thakur KT, Lyons JL, Smith BR, et al. Stroke in HIV-infected African Americans: a retrospective cohort study. J Neurovirol 2016; 22: 50-55.

40. Bermel C, Spüntrup E, Fink G, et al. Stroke in an adult with HIV infection due to carotid artery stenosis successfully treated with steroids: HIV-associated arteritis? J Neurol 2009; 256: 1563-1565.

41. Taylor C, Varma A, Herwadkar A, et al. Successful reversal of threatening carotid artery occlusion in HIV-associated non-aneurysmal vasculitis. Int J STD AIDS 2008; 19: 141-142. 
42. Assallum H, Alkayem M, Shabarek N. HIV infection and acute stroke: a case report and a review of the literature. Case Rep Med 2013; 2013: 892054.

43. Riddler SA, Smit E, Cole SR, et al. Impact of HIV infection and HAART on serum lipids in men. JAMA 2003; 289: 2978-2982.

44. Schillaci G, De Socio GV, Pirro M, et al. Impact of treatment with protease inhibitors on aortic stiffness in adult patients with human immunodeficiency virus infection. Arterioscler Thromb Vasc Biol 2005; 25: 2381-2385.

45. Torriani FJ, Komarow L, Parker RA, et al. Endothelial function in human immunodeficiency virus-infected antiretroviral-naive subjects before and after starting potent antiretroviral therapy: The ACTG (AIDS Clinical Trials Group) Study 5152s. J Am Coll Cardiol 2008; 52: 569-576.

46. Murphy R, Costagliola D. Increased cardiovascular risk in HIV infection: drugs, virus and immunity. AIDS 2008; 22: 1625-1627.

47. Otedo A, Oyoo G, Obondi J, et al. Vasculitis in HIV: report of eight cases. East Afr Med J 2005; 82: 656-659.

48. Quijano G, Drut R. Atherosclerosis and central adiposity in a pediatric patient with AIDS treated with HAART: autopsy findings. Pediatr Dev Pathol 2006; 9: 474-479.

49. Blann A, Seigneur M, Constans J, et al. Soluble P-selectin, thrombocytopenia and von Willebrand factor in HIV infected patients. Thromb Haemost 1997; 77: 1221-1222.

50. Karmochkine M, Ankri A, Calvez V, et al. Plasma hypercoagulability is correlated to plasma HIV load. Thromb Haemost 1998; 80: 208-209.

51. Erbe M, Rickerts V, Bauersachs RM, et al. Acquired protein C and protein $\mathrm{S}$ deficiency in HIV-infected patients. Clin Appl Thromb Hemost 2003; 9: 325-331.

52. Mizusawa H, Hirano A, Llena J, et al. Cerebrovascular lesions in acquired immune deficiency syndrome (AIDS). Acta Neuropathol 1988; 76: 451-457.

53. Cohen JE, Eger K, Montero A, et al. Rapid spontaneous resolution of acute subdural hematoma and HIV related cerebral atrophy: Case report. Surg Neurol 1998; 50: 241-244.

54. Roquer J, Palomeras E, Knobel H, et al. Intracerebral haemorrhage in AIDS. Cerebrovasc Dis 1998; 8: 222-227.

55. Thakur KT, Lyons JL, Smith BR, et al. Stroke in HIV-infected African Americans: a retrospective cohort study. J Neurovirol 2016; 22 : 50-55.

56. Jordan BD, Navia BA, Petito C, et al. Neurological syndromes complicating AIDS. Cancer and AIDS. Karger Publishers, 1985; pp. 82-87.

57. Afsari K, Frank J, Vaksman Y, et al. Intracranial venous sinus thrombosis complicating AIDS-associated nephropathy. AIDS Reader 2003; 13: 143-148.

58. Alomari A, Jiarakongmun P, Dejthevaporn C, et al. Multiple symptomatic cerebral arteriovenous malformations in a patient with HIV. Am J Neuroradiol 2009; 30: 1273-1274.

59. Park YD, Belman AL, Kim TS, et al. Stroke in pediatric acquired immunodeficiency syndrome. Ann Neurol 1990; 28: 303-311.

60. Fulmer BB, Dillard SC, Musulman EM, et al. Two cases of cerebral aneurysms in HIV+ children. Pediatr Neurosurg 1998; 28: 31-34.

61. Shah SS, Zimmerman RA, Rorke LB, et al. Cerebrovascular complications of HIV in children. Am J Neuroradiol 1996; 17: 1913-1917.

62. Narayan P, Samuels OB, Barrow DL. Stroke and pediatric human immunodeficiency virus infection. Pediatr Neurosurg 2002; 37: 158-163.

63. Kure K, Park YD, Kim TS, et al. Immunohistochemical localization of an HIV epitope in cerebral aneurysmal arteriopathy in pediatric acquired immunodeficiency syndrome (AIDS). Pediatr Pathol 1989; 9: 655-667.

64. Schieffelin JS, Williams PL, Djokic D, et al. Central nervous system vasculopathy in HIV-infected children enrolled in the pediatric AIDS Clinical Trials Group 219/219C Study. J Pediatr Infect Dis Soc 2013; 2: 50-56.
65. Kabus D, Greco MA. Arteriopathy in children with AIDS: microscopic changes in the vasa vasorum with gross irregularities of the aortic intima. Pediatr Pathol 1991; 11: 793-795.

66. Joshi VV, Pawel B, Connor E, et al. Arteriopathy in children with acquired immune deficiency syndrome. Pediatr Pathol 1987; 7: 261-275.

67. Chetty R. Vasculitides associated with HIV infection. J Clin Pathol 2001; 54: 275-278.

68. Marks C, Kuskov S. Pattern of arterial aneurysms in acquired immunodeficiency disease. World J Surg 1995; 19: 127-132.

69. Katsetos CD, Fincke JE, Legido A, et al. Angiocentric CD3+ T-cell infiltrates in human immunodficiency virus type 1-associated central nervous system disease in children. Clin Diagn Lab Immunol 1999; 6: 105-114.

70. Simpson DM, Berger JR. Neurologic manifestations of HIV infection. Med Clin North Am 1996; 80: 1363-1394.

71. Sharer LR, Saito Y, Da Cunha A, et al. In situ amplification and detection of HIV-1 DNA in fixed pediatric AIDS brain tissue. Hum Pathol 1996; 27: 614-617.

72. Leeuwis JW, Wolfs TF, Braun KP. A child with HIV-associated transient cerebral arteriopathy. AIDS 2007; 21: 1383-1384.

73. Connor MD. Treatment of HIV associated cerebral vasculopathy. J Neurol Neurosurg Psychiatry 2009; 80: 831.

74. Cole JW, Pinto AN, Hebel JR, et al. Acquired immunodeficiency syndrome and the risk of stroke. Stroke 2004; 35: 51-56.

75. Izbudak I, Chalian M, Hutton N, et al. Perinatally HIV-infected youth presenting with acute stroke: Progression/evolution of ischemic disease on neuroimaging. J Neuroradiol 2013; 40: 172-180.

76. Maggi P, Perilli F, Lillo A, et al. An ultrasound-based comparative study on carotid plaques in HIV-positive patients vs. atherosclerotic and arteritis patients: atherosclerotic or inflammatory lesions? Coron Artery Dis 2007; 18: 23-29. 